\title{
International Agency for Research on Cancer
}

\section{IARC: Outdoor air pollution a leading environmental cause of cancer deaths}

Lyon/Geneva, 17 October 2013 - The specialized cancer agency of the World Health Organization, the International Agency for Research on Cancer (IARC), announced today that it has classified outdoor air pollution as carcinogenic to humans (Group 1).

After thoroughly reviewing the latest available scientific literature, the world's leading experts convened by the IARC Monographs Programme concluded that there is sufficient evidence that exposure to outdoor air pollution causes lung cancer (Group 1). They also noted a positive association with an increased risk of bladder cancer.

Particulate matter, a major component of outdoor air pollution, was evaluated separately and was also classified as carcinogenic to humans (Group 1).

The IARC evaluation showed an increasing risk of lung cancer with increasing levels of exposure to particulate matter and air pollution. Although the composition of air pollution and levels of exposure can vary dramatically between locations, the conclusions of the Working Group apply to all regions of the world.

\begin{abstract}
A major environmental health problem
Air pollution is already known to increase risks for a wide range of diseases, such as respiratory and heart diseases. Studies indicate that in recent years exposure levels have increased significantly in some parts of the world, particularly in rapidly industrializing countries with large populations. The most recent data indicate that in 2010, 223000 deaths from lung cancer worldwide resulted from air pollution. ${ }^{2}$
\end{abstract}

\section{The most widespread environmental carcinogen}

"The air we breathe has become polluted with a mixture of cancer-causing substances," says Dr Kurt Straif, Head of the IARC Monographs Section. "We now know that outdoor air pollution is not only a major risk to health in general, but also a leading environmental cause of cancer deaths."

The IARC Monographs Programme, dubbed the "encyclopaedia of carcinogens", provides an authoritative source of scientific evidence on cancer-causing substances and exposures. In the past, the Programme evaluated many individual chemicals and specific mixtures that occur in outdoor air pollution. These included diesel engine exhaust, solvents, metals, and dusts. But this is the first time that experts have classified outdoor air pollution as a cause of cancer.

"Our task was to evaluate the air everyone breathes rather than focus on specific air pollutants," explains Dr Dana Loomis, Deputy Head of the Monographs Section. "The results from the reviewed studies point in the same direction: the risk of developing lung cancer is significantly increased in people exposed to air pollution."

\section{IARC Monographs evaluations}

Volume 109 of the IARC Monographs is based on the independent review of more than 1000 scientific papers from studies on five continents. The reviewed studies analyse the carcinogenicity of various pollutants present in outdoor air pollution, especially particulate matter and transportation-related pollution. The evaluation is driven by findings from large epidemiologic studies that included millions of people living in Europe, North and South America, and Asia.

\footnotetext{
${ }^{1}$ Please note that the summary evaluation will be published by The Lancet Oncology online on Thursday 24 October 2013

${ }^{2}$ http://www.iarc.fr/en/publications/books/sp161/index.php
} 


\section{IARC: Outdoor air pollution a leading environmental cause of cancer deaths}

The predominant sources of outdoor air pollution are transportation, stationary power generation, industrial and agricultural emissions, and residential heating and cooking. Some air pollutants have natural sources, as well.

"Classifying outdoor air pollution as carcinogenic to humans is an important step," stresses IARC Director Dr Christopher Wild. "There are effective ways to reduce air pollution and, given the scale of the exposure affecting people worldwide, this report should send a strong signal to the international community to take action without further delay."

For more information, please contact

Véronique Terrasse, Communications Group, or at +33 (0) 645284952 ;

or Dr Nicolas Gaudin, IARC Communications

The International Agency for Research on Cancer (IARC) is part of the World Health Organization. Its mission is to coordinate and conduct research on the causes of human cancer, the mechanisms of carcinogenesis, and to develop scientific strategies for cancer control. The Agency is involved in both epidemiological and laboratory research and disseminates scientific information through publications, meetings, courses, and fellowships. If you wish your name to be removed from our press release emailing list, please write to com@iarc.fr. 


\section{IARC: Outdoor air pollution a leading environmental cause of cancer deaths}

\section{Annexes}

\section{Evaluation groups - Definitions}

Group 1: The agent is carcinogenic to humans.

This category is used when there is sufficient evidence of carcinogenicity in humans. Exceptionally, an agent may be placed in this category when evidence of carcinogenicity in humans is less than sufficient but there is sufficient evidence of carcinogenicity in experimental animals and strong evidence in exposed humans that the agent acts through a relevant mechanism of carcinogenicity.

\section{Group 2.}

This category includes agents for which, at one extreme, the degree of evidence of carcinogenicity in humans is almost sufficient, as well as those for which, at the other extreme, there are no human data but for which there is evidence of carcinogenicity in experimental animals. Agents are assigned to either Group 2A (probably carcinogenic to humans) or Group 2B (possibly carcinogenic to humans) on the basis of epidemiological and experimental evidence of carcinogenicity and mechanistic and other relevant data. The terms probably carcinogenic and possibly carcinogenic have no quantitative significance and are used simply as descriptors of different levels of evidence of human carcinogenicity, with probably carcinogenic signifying a higher level of evidence than possibly carcinogenic.

- Group 2A: The agent is probably carcinogenic to humans.

This category is used when there is limited evidence of carcinogenicity in humans and sufficient evidence of carcinogenicity in experimental animals. In some cases, an agent may be classified in this category when there is inadequate evidence of carcinogenicity in humans and sufficient evidence of carcinogenicity in experimental animals and strong evidence that the carcinogenesis is mediated by a mechanism that also operates in humans. Exceptionally, an agent may be classified in this category solely on the basis of limited evidence of carcinogenicity in humans. An agent may be assigned to this category if it clearly belongs, based on mechanistic considerations, to a class of agents for which one or more members have been classified in Group 1 or Group 2A.

- Group 2B: The agent is possibly carcinogenic to humans.

This category is used for agents for which there is limited evidence of carcinogenicity in humans and less than sufficient evidence of carcinogenicity in experimental animals. It may also be used when there is inadequate evidence of carcinogenicity in humans but there is sufficient evidence of carcinogenicity in experimental animals. In some instances, an agent for which there is inadequate evidence of carcinogenicity in humans and less than sufficient evidence of carcinogenicity in experimental animals together with supporting evidence from mechanistic and other relevant data may be placed in this group. An agent may be classified in this category solely on the basis of strong evidence from mechanistic and other relevant data.

Group 3: The agent is not classifiable as to its carcinogenicity to humans.

This category is used most commonly for agents for which the evidence of carcinogenicity is inadequate in humans and inadequate or limited in experimental animals.

Exceptionally, agents for which the evidence of carcinogenicity is inadequate in humans but sufficient in experimental animals may be placed in this category when there is strong evidence that the mechanism of carcinogenicity in experimental animals does not operate in humans.

Agents that do not fall into any other group are also placed in this category.

An evaluation in Group 3 is not a determination of non - carcinogenicity or overall safety. It often means that further research is needed, especially when exposures are widespread or the cancer data are consistent with differing interpretations.

\section{Group 4: The agent is probably not carcinogenic to humans.}

This category is used for agents for which there is evidence suggesting lack of carcinogenicity in humans and in experimental animals. In some instances, agents for which there is inadequate evidence of 


\section{IARC: Outdoor air pollution a leading environmental cause of cancer deaths}

carcinogenicity in humans but evidence suggesting lack of carcinogenicity in experimental animals, consistently and strongly supported by a broad range of mechanistic and other relevant data, may be classified in this group.

\section{Evidence for studies in humans - Definition}

As shown previously, the evidence relevant to carcinogenicity is evaluated using standard terms. For studies in humans, evidence is defined into one of the following categories:

Sufficient evidence of carcinogenicity: The Working Group considers that a causal relationship has been established between exposure to the agent and human cancer. That is, a positive relationship has been observed between the exposure and cancer in studies in which chance, bias and confounding could be ruled out with reasonable confidence. A statement that there is sufficient evidence is followed by a separate sentence that identifies the target organ(s) or tissue(s) where an increased risk of cancer was observed in humans. Identification of a specific target organ or tissue does not preclude the possibility that the agent may cause cancer at other sites.

Limited evidence of carcinogenicity: A positive association has been observed between exposure to the agent and cancer for which a causal interpretation is considered by the Working Group to be credible, but chance, bias or confounding could not be ruled out with reasonable confidence.

Inadequate evidence of carcinogenicity: The available studies are of insufficient quality, consistency or statistical power to permit a conclusion regarding the presence or absence of a causal association between exposure and cancer, or no data on cancer in humans are available.

Evidence suggesting lack of carcinogenicity: There are several adequate studies covering the full range of levels of exposure that humans are known to encounter, which are mutually consistent in not showing a positive association between exposure to the agent and any studied cancer at any observed level of exposure. The results from these studies alone or combined should have narrow confidence intervals with an upper limit close to the null value (e.g. a relative risk of 1.0). Bias and confounding should be ruled out with reasonable confidence, and the studies should have an adequate length of follow - up. A conclusion of evidence suggesting lack of carcinogenicity is inevitably limited to the cancer sites, conditions and levels of exposure, and length of observation covered by the available studies. In addition, the possibility of a very small risk at the levels of exposure studied can never be excluded.

In some instances, the above categories may be used to classify the degree of evidence related to carcinogenicity in specific organs or tissues. 